\title{
Decompressive craniectomy in patients with refractory intracranial hypertension after traumatic brain injury: A systematic review and meta-analysis of current evidence
}

\author{
G.Tsaousi', PG. Sergi'², L. Marocchi², C. Pourzitaki', A. Santoro ${ }^{3}$, F. Bilotta². \\ ${ }^{1}$ Department of Anesthesiology and ICU, Aristotle University of Thessaloniki, Faculty of Medicine, Thessaloniki, Greece \\ 2Department of Anesthesiology, University of Rome "La Sapienza, Rome, Italy \\ 3Department of Neurosurgery, University of Rome "La Sapienza, Rome, Italy
}

\section{Background and Goal of Study}

Intracranial hypertension constitutes a common clinical entity after traumatic brain injury (TBI), addressed to divergent pathologies such as mass effect due to intracranial hematomas and contusions, diffuse brain swelling, or hydrocephalus. Decompressive craniectomy (DC) to control refractory intracranial hypertension in patients with TBI has been listed as possible but controversial therapeutic approach in the latest version of TBI management guidelines. This systematic review and meta-analysis aims to report current clinical evidence, published between 2011 and 2017- a timeline in which the most robust evidence occurred - on efficacy and safety of DC compared to standard care to treat intractable intracranial pressure after TBI.

\section{Materials and Methods}

A database search (PubMed, EMBASE, CENTRAL and International Web of Science) from 2011 to 2017 was conducted to identify randomized clinical trials (RCTs) and observational studies pertinent to DC compared to standard care after severe TBI. The primary outcomes were mortality and functional outcome upon hospital discharge and at 6 and 12 months after intervention, whereas intracranial pressure (ICP) control, hospitalization data and occurrence of DC-related adverse events or systemic side-effects served as secondary outcomes. Selected full papers were critically appraised and quality-assessed, using the Jadad scale and the ROBINS-1 tool for RCTs and observational cohort studies, respectively. The bias risk in each study was judged by Cochrane Collaboration Risk of Bias Tool. The results of RCTs and observational studies were pooled and weighted separately and then together, using Review Manager. We computed risk ratios (RRs) with 95\% confidence intervals (Cls) for dichotomous data. Between-study heterogeneity was assessed with the Cochrane $Q$ test. Within-group heterogeneity was quantified using the $\mathrm{I}^{2}$ statistic.

\section{Results}

A total of 9533 records were retrieved from database search and 1055 were screened after filtering. Out of these, 24 were excluded as duplicates, whilst one study was considered of inappropriate methodology. Finally, 5 trials including 3 RCTs and 2 observational studies, enrolling 1269 patients, were selected for qualitative analysis, among which one deemed unsuitable to be included in meta-analysis. [Figure 1]. Most of the retrieved studies were of high risk of bias and rather low quality. Mortality and functional outcome constituted the combined end-points in 4 studies, whilst in one trial the main point of interest was mortality. DC-treated patients showed a significant reduction in their overall mortality $(\mathrm{RR}, 0.57 ; 95 \% \mathrm{Cl}, 0.5-0.66$; $\mathrm{P}<0.001 ;\left.\right|^{2}=17 \%$ ) with no profound beneficial effect on their functional outcome (RR, $0.89 ; 95 \% \mathrm{Cl}, 0.78-1.02 ; \mathrm{P}=0.09 ; \mathrm{I}^{2}=58 \%$ ) compared to those receiving standard care [Figure 2]. However, a notable improvement on functional outcome was recorded only at 12 months after intervention (RR, $0.80 ; 95 \% \mathrm{Cl}, 0.70-0.92 ; \mathrm{P}=0.001 ; \mathrm{I}^{2}=0 \%$ ) [Figure $2 \mathrm{~B}$ ]. Although, $\mathrm{DC}$ yielded a positive impact on ICP control and duration of ICU stay, its effect in reducing the length of hospitalization was less clear. In terms of safety, DC was associated with an augmented risk of adverse effects.

\section{Conclusion}

Based on current evidence, DC emerges as a superior practice to standard care for intracranial hypertension control, with a subsequent short- to longterm survival benefit in TBI patients. Available evidence also suggests a trend towards to late functional outcome improvement; yet no definite conclusion can be drawn due to limited quantity and considerable heterogeneity of available data.
Figure 1. Flow diagram showing the results of the search and reasons for studies exclusion.

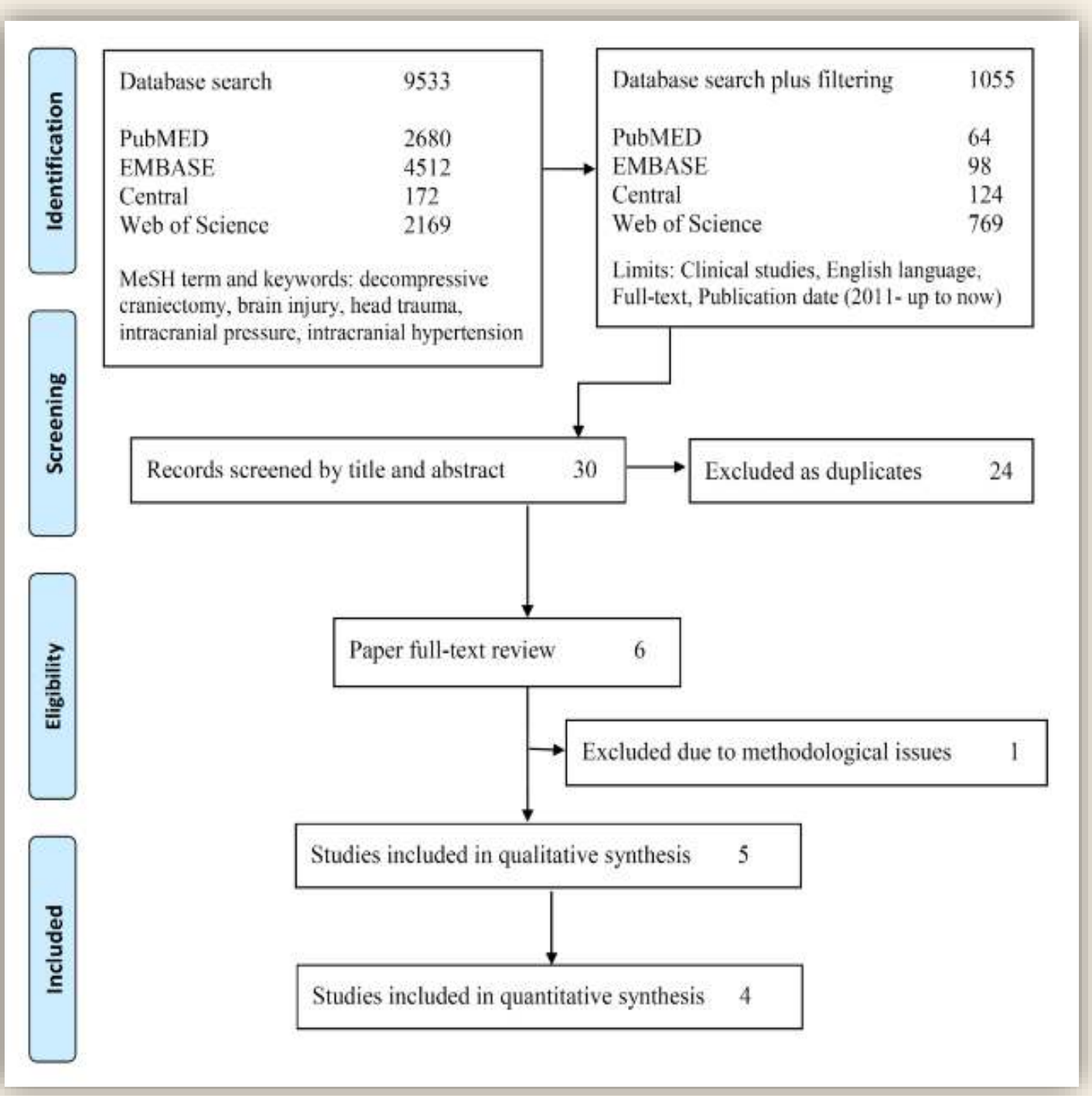

Figure 2. Forrest plot showing the pooled estimate of the risk of mortality and adverse functional outcome of DC to standard care.

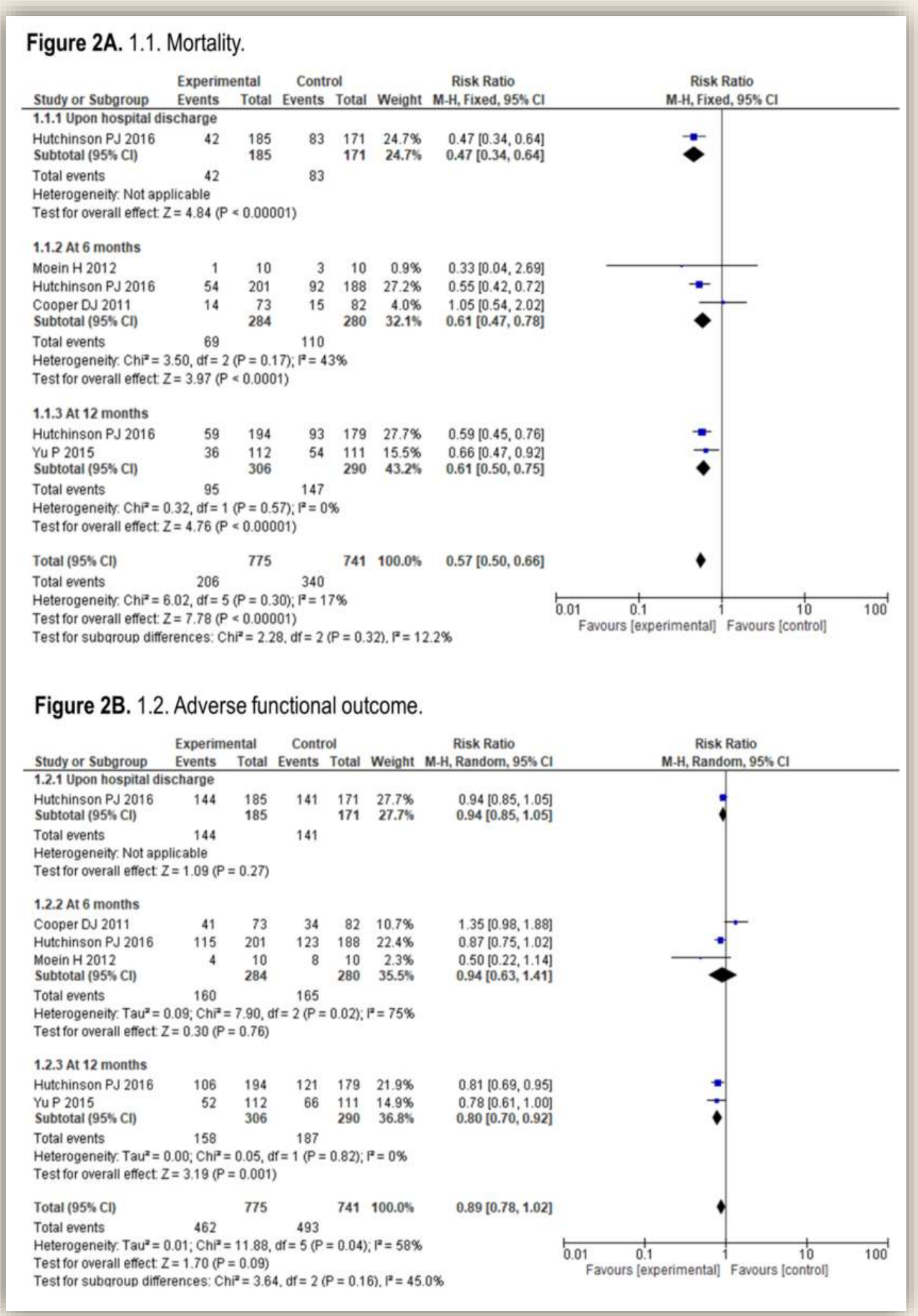

